\title{
Roles of Diffusible Signals in Communication Among Plant-Associated Bacteria
}

\author{
Leland S. Pierson III and Elizabeth A. Pierson
}

Department of Plant Sciences, Division of Plant Pathology and Microbiology, The University of Arizona, Tucson 85721.

\begin{abstract}
Pierson, L. S., III, and Pierson, E. A. 2007. Roles of diffusible signals in communication among plant-associated bacteria. Phytopathology 97:227232 .

In nature, Pseudomonas species compete and co-exist in mixed communities with a diversity of prokaryotic and eukaryotic micro- and macroorganisms. Many bacteria produce various signals that control gene expression and thus contribute to specific bacterial behaviors and coordinate essential functions with other members of the community. The best-studied signaling compounds are $\mathrm{N}$-acyl-homoserine lactones (AHLs), which are involved in quorum sensing (QS) regulation and are

produced by a diverse range of bacterial taxa. To date, research on QS has focused on how signals control gene expression in the bacterial cell and the role of these signals in positive and negative communication among different groups of organisms. Additionally, mechanisms for AHL decay and AHL utilization as sole carbon/energy sources have been identified. Some host organisms produce compounds that can mimic AHLs, and some bacterial signals can influence host gene expression. Thus, interkingdom communication may be more widespread than previously believed. Our current understanding of individual, community and bacterial-host interactions is still in its infancy and there are many exciting discoveries yet to be made.
\end{abstract}

All host-associated bacteria, whether pathogenic, beneficial, or commensal, must integrate into the physical, chemical, nutritional, and biotic environment of the host organism. Central to this integration is the appropriate expression of bacterial traits essential for each of the various stages of host interaction. Traits include those involved in host detection, motility toward the host, colonization, nonactivation or avoidance of host defenses, growth, and reproduction. Successful interaction with the host requires precise activation and repression of genes at the proper time (temporal regulation) and at the proper location (spatial regulation). Bacteria have evolved numerous sensory systems that allow them to control gene expression in response to various environmental parameters and specific chemical signals. One subset of these sensory genes has evolved to respond to the presence or absence of specific diffusible signal molecules. These communication networks represent ecological control points that direct complex changes in bacterial gene expression patterns and, by so doing, the outcome of bacterial-host interactions. By understanding these sensory systems and the mechanisms and roles of microbial communication, we may be able to manipulate bacterial-host interactions for improved biological control.

Recently, there has been an explosion of information regarding communication within and between bacterial populations. Other recent areas of research include the effect of bacterial signals on gene expression in their eukaryotic hosts and the effect of the host on bacterial signaling. Although detailed examination of all aspects of microbial signaling is beyond the scope of this review, specific aspects of communication among bacteria and between bacteria and their hosts via diffusible signals are discussed. While focusing on the genus Pseudomonas, examples are provided using

Corresponding author: L. S. Pierson III; E-mail address: 1sp@u.arizona.edu

DOI: 10.1094/PHYTO-97-2-0227

(c) 2007 The American Phytopathological Society other bacteria to illustrate specific points and potential areas for future research.

Communication signals. Communication can be defined as the process of exchanging information, usually via a common system of symbols. Functions regulated by communication include cell co-aggregation via specific receptors, the production of specific secondary metabolites, and the exchange of genetic material. For plant-associated bacteria, communication can play key roles in community interactions and temporal and spatial aspects of host interactions. In this paper, we will focus on some of the known roles of diffusible signal molecules in communication. The diffusible signals that will be considered in this article include $\mathrm{N}$ acyl-homoserine lactones (AHLs), autoinducer 2 (AI-2), 2-heptyl3-hydroxy-4-quinoline (PQS), and eukaryotic signal mimics.

AHLs. In gram-negative bacteria, the best-studied diffusible signals are the AHLs (Fig. 1A). This class of signal consists of a conserved homoserine lactone ring moiety connected to a fatty acyl side chain $(13,17,19)$. The length of the side chains can vary, as well as the types and number of side chain modifications. For Pseudomonas species, AHL signals have been shown to regulate the expression of genes that encode traits important in pathogenicity, competition, or survival within a bacterial population. AHL-mediated regulation is most commonly referred to as quorum sensing (QS) because, in the majority of cases, a certain bacterial population density (a quorum) is required for gene expression to occur. The simplest model for QS regulation involves two proteins and was first elucidated in the marine symbiont Vibrio fischeri $(16,19)$. The first protein is an AHL synthase, encoded by a gene referred to as an $I$ gene, that converts cellular precursors into one or more AHLs. These genes are classically designated by the phenotype regulated (i.e., in V. fischeri the gene encoding the AHL synthase is named luxI since bioluminescence is the trait regulated by $\mathrm{QS})$. The second is a regulatory protein $(\mathrm{R}$ protein), encoded by a gene referred to as an $R$ gene, which is required for the activation (or repression) of specific promoters. At low cell density, the AHL signals generated by the AHL 
synthase diffuse out of the producing cell along a concentration gradient. As cell numbers increase, the concentration of AHL signals around the cell also increases. When the concentration of intracellular AHL reaches a threshold level, the signal interacts with the $\mathrm{R}$ protein. Interaction of the AHL with the $\mathrm{R}$ protein increases the affinity of the $\mathrm{R}$ protein for specific sequences in the promoter regions of genes under $\mathrm{QS}$ control, resulting in $\mathrm{R}$ protein binding and activation (or repression) of target genes (16,38, 52). Although this form of regulation is most commonly termed QS, a single bacterial cell can have QS-regulated genes activated in the presence of sufficient AHL signal. Thus, it is the concentration of AHL, not the number of bacteria per se, that determines gene expression patterns.

The phenomenon known as QS was identified first in a single bacterial population, and therefore was originally termed autoinduction (30). It soon became apparent that communication via AHL signals occurs between related and unrelated bacterial species. One example of this type of cross-communication was demonstrated using Pseudomonas chlororaphis (aureofaciens) strain 30-84 $(34,37,48)$. P . chlororaphis produces three broadspectrum phenazine antibiotics, a yellow phenazine-1-carboxylic acid and two orange 2-hydroxy-derivatives (36). The phenazine biosynthetic locus, composed of eight genes in a single operon ( $p h z X Y F A B C D O)(27,35)$, is regulated directly by the PhzR/PhzI QS system. PhzI is an AHL synthase that produces hexanoyl homoserine lactone (HHL), and PhzR is the transcriptional regulator. Inactivation of phzI in strain 30-84 results in loss of HHL production and orange pigmentation, which is a marker for phenazine production in strain 30-84 (48). Phenazine production can be restored by the addition of exogenous HHL.

A random collection of wheat rhizosphere bacteria was screened for the capacity to complement a phzI mutant of strain 30-84 by spotting each on a lawn of mutant bacteria. Approximately $8 \%$ of strains restored phenazine production based on restoration of orange pigmentation. This demonstrated that these strains produced diffusible signals recognized by the phzI mutant in vitro. The ability to restore phenazine gene expression to a phzI mutant also was demonstrated in situ on wheat roots using a strain 30-84 $p h z I^{-}, p h z B:: i n a Z$ genomic reporter (33). This strain expresses ice nucleation activity as a reporter for $p h z$ gene expression. Loss of phzI resulted in a 1,000-fold decrease in ice nucleation activity compared with that of the $p h z I^{+}$strain. Several wheat rhizosphere

A.

B.

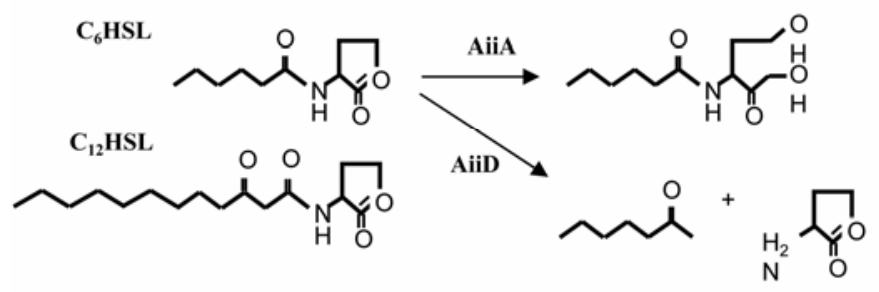

C.
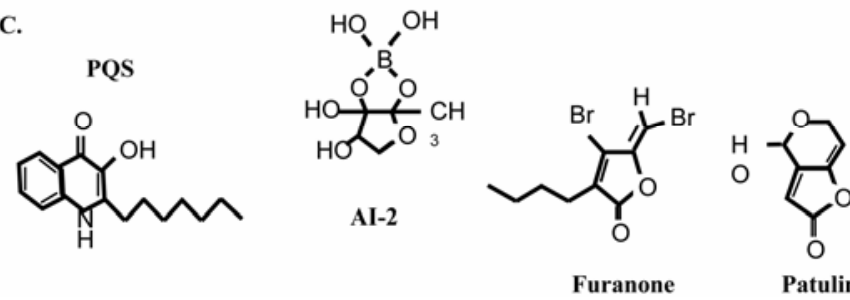

Fig. 1. Diffusible signals produced by bacteria and fungi. A, Two representative $\mathrm{N}$-acyl homoserine lactone (AHL) structures are shown, $\mathrm{C}_{6} \mathrm{HSL}$ and $\mathrm{C}_{12} \mathrm{HSL}$. B, Degradation products generated by the Bacillus spp. AHL lactonase protein AiiA and the Ralstonia spp. AHL acylase protein AiiD. C, Generic structures of the 4-quinoline signal PQS, autoinducer 2 (AI-2), furanones, and patulin. strains restored ice nucleation activity by the reporter strain to wild-type levels on roots in soil, demonstrating QS was involved in phenazine production on roots and that communication occurred between bacterial populations via AHL signals. A second example of positive communication between closely related bacteria was demonstrated using a P. putida F117 ppuI AHL synthase mutant. This mutant contained a reporter plasmid with a green fluorescent protein (GFP) gene, which encoded an unstable GFP, fused to the $P$. aeruginosa las promoter (41). GFP fluorescence was only detected in the presence of $P$. putida strain Z2D (an AHL producer) on tomato roots. Recently, Williamson et al. (47) developed a rapid intracellular screening method (METREX, for metabolite-regulated expression) to identify clones from a metagenomic library constructed from microbes in a permafrost-free Alaskan soil that produced small bioactive molecules that activated or repressed QS-regulated genes. This assay identified a higher frequency of QS inducer genes in the metagenomic library than were identified by standard cultured bacterial libraries from the same source.

Autoinducer 2. Autoinducer 2 (AI-2) is a family of closely related furanosyl-borate diester molecules (Fig. 1C). It was first described in 1997 and was hypothesized to be a universal bacterial signal $(2,42,49,50)$. AI- 2 is synthesized by the products of the pfs and luxS genes. Although AI-2 is produced by over 30 gram-positive and gram-negative bacteria, the role of AI-2 in bacterial signaling currently is unclear $(44,49)$. It has been shown to be involved in the activated methyl cycle in bacteria, and it also can be used as a nutrient source by bacteria.

The best-characterized AI-2 regulatory system is in bioluminescent Vibrio species, including $V$. harveyi. In the absence of a sufficient AI-2 concentration, a signal cascade mechanism involving LuxP, LuxQ, and LuxU results in the phosphorylation of LuxO. Phosphorylation of LuxO, in conjunction with sigma factor RpoN $\left(\sigma^{54}\right)$, results in activation of a small regulatory RNA that causes luxR mRNA to be destabilized. The net result is no production of the LuxR protein or expression of the bacterium's bioluminescence operon (lux operon). When sufficient AI-2 is present, the phosphorylation cascade reverses, resulting in dephosphorylation of LuxO, lack of expression of the small regulatory RNA, and expression of the lux operon. Thus, the $V$. harveyi LuxR protein does not require AHL to function, unlike the classic LuxR QS systems (e.g., V. fischeri), which depend on AHL signals to function.

Although no LuxS homologue has been identified in Pseudomonas spp., a potential role for AI-2 in communication in $P$. aeruginosa has been suggested by Duan et al. (15). P. aeruginosa is an opportunistic pathogen that is a primary cause of increased morbidity and mortality in patients suffering from cystic fibrosis. It colonizes patient lungs and utilizes two hierarchical QS systems to regulate virulence gene expression. However, $P$. aeruginosa is a member of a complex oropharyngeal (OF) bacterial community in the lungs. The presence of this OF community apparently has little effect on lung health and no effect on the population size of $P$. aeruginosa, but enhances $P$. aeruginosa virulence as measured in a murine model (15). Of particular interest is the fact that the majority of these OF bacteria are gram-positive species that do not synthesize AHLs, but do synthesize AI-2. AI-2 can be readily isolated from patient sputum samples. A promoter trap library of $P$. aeruginosa indicated that the presence of OF bacterial strains altered the expression of $\approx 4 \%$ of the $P$. aeruginosa genome (15). Expression of 6 of the 21 promoters examined was increased by the addition of purified AI-2. Genes increased in expression by AI-2 included several involved in virulence, motility, and biosurfactant production. These results indicate the exciting possibility that $P$. aeruginosa is capable of "listening" to other bacterial communication systems. This is similar to what was found in Salmonella typhimurium (1). Here strains of S. typhimurium were identified that responded to the presence of AHL signals although 
they lacked the ability to synthesize AHLs themselves. The ecological impact of this eavesdropping is as yet unclear, but it does indicate the complexity of communication signals and potential code-breaking that occurs in microbial communities.

PQS. In addition to AHL-mediated QS regulation, P. aeruginosa contains a second interconnected QS signaling pathway that also is involved in the expression of virulence determinants and other secondary metabolites (32). The signal PQS (2-heptyl-3hydroxy-4-quinoline) (Fig. 1C) is synthesized by the condensation of anthranilate and $\beta$-keto-(do)decanoic acids by the products of the pqsABCDE operon (4). Interestingly, PQS synthesis appears to be controlled by competition between the LasR/LasI and RhlR/RhlI QS systems (7,28). Production of PQS occurs during late exponential to stationary growth phases and is believed to be important for biofilm development (8), virulence in plants (5), and cystic fibrosis development in humans (20).

Although PQS was first detected in $P$. aeruginosa culture supernatants, it was unclear how this hydrophobic molecule was transported out of the cell. Recently, Mashburn and Whiteley (25) showed that in $P$. aeruginosa PQS mediates its own packaging into membrane vesicles. These membrane vesicles can move between cells within the population and between $P$. aeruginosa and other gram-negative and gram-positive bacteria. These results suggest that $P$. aeruginosa possesses a signal trafficking system analogous to eukaryotic organisms that can coordinate bacterial group behaviors.

Signal interference. Bacterial communication interference. Communication among bacterial populations appears to be prevalent in nature. The ability to disrupt or interfere with these communications also appears to be widespread and can alter the expression of specific traits. For example, we hypothesized that because some members of the wheat rhizosphere community could restore phenazine production in a phzI mutant of P. chlororaphis (described above), then perhaps a second subpopulation could inhibit phenazine production. If true, this could explain some of the observed inconsistent results with biological control agents during field trials. By spotting our wheat rhizosphere library on wild-type orange $P$. chlororaphis strain 30-84, we identified a subset of strains $(\approx 7 \%)$ that blocked phenazine production, as indicated by the development of a white halo surrounding colonies of these strains (29). A genomic phzB::lacZ reporter strain, which expressed $\beta$-galactosidase in place of phenazines, was used to show that inhibition of phenazine production occurred at the level of gene expression. Recently, using a negative signaling reporter plasmid in which PhzR binding repressed the expression of another reporter gene, we verified that negative signaling occurs in situ on wheat roots $(\mathrm{E}$. A. Pierson, J. Morello, and L. S. Pierson, III, unpublished data).

Dong et al. (9) isolated a Bacillus spp. (240B1) from soil in which AHL signals are degraded. This strain contained the gene aiiA, which encodes an AHL lactonase that inactivates AHL signals by hydrolysis of the homoserine lactone ring (Fig. 1B). The plant pathogen Erwinia carotovora causes soft-rotting disease on plants due to production of tissue macerating enzymes, such as pectinases and cellulases, under QS control. Construction of a transgenic E. carotovora strain expressing aiiA resulted in the inability to induce soft-rot symptoms when inoculated on Chinese cabbage, demonstrating that AHL degradation in the pathogen blocked pathogenicity. Recently, a Ralstonia spp. was isolated that contained an aiiD gene encoding an AHL acylase (Fig. 1B), which cleaves the fatty acyl moiety from the homoserine lactone ring (22). Transgenic $P$. aeruginosa strains expressing aiiD also were blocked in the expression of QS-regulated virulence traits. The opportunistic pathogen $P$. aeruginosa PAO1 contains a homologue of the Ralstonia AHL acylase, termed $p v d Q$. P. aeruginosa engineered to express the $p v d Q$ gene constitutively failed to accumulate the long chain AHL signal. However, inactivation of $p v d Q$ did not result in the inability of $P$. aeruginosa to grow on long chain AHLs, indicating the mutants were still able to degrade AHL signals themselves, although slowly.

The production of AHL signals in the bacterial environment is necessary for the expression (or repression) of QS-regulated traits. However, once signals accumulate, there must be mechanisms for resetting the system by reducing their concentrations. Signal degradation via AHL degrading enzymes represents one mechanism for this. P. aeruginosa strain PAI-1, isolated from turf soil, was shown to utilize the long acyl side chain AHL 3-oxo$\mathrm{C}_{12} \mathrm{HSL}$ as its sole energy source $(21,45)$. It could not utilize short side chain AHLs for growth. Subsequent work using various soil samples indicated rapid degradation of ${ }^{14} \mathrm{C}-\mathrm{C}_{6} \mathrm{HSL}$ from soil (45). Steam-sterilized soil did not demonstrate this effect, implicating a biological component. Metabolism of the AHL signal was indicated further by the release of volatile ${ }^{14} \mathrm{CO}_{2}$. It appears that there is a community-wide ability to rapidly degrade AHL signals in the environment, and it has been hypothesized that this degradation is an essential component of QS regulation by quieting signal crosstalk or serving to reset QS systems.

Eukaryotic communication interference. Until recently, the eukaryotic host was considered a "silent" partner in bacterial QS systems. However, it is becoming increasingly clear that the host has a significant influence on bacterial communication, both by interfering with or activating QS regulatory systems. For example, the marine macroalgae Delisea pulchra has been shown to produce over 25 furanone compounds that serve as AHL mimic compounds (24). Furanones are chemically similar to AHLs, and their presence blocks many QS-regulated traits (Fig. 1C). Addition of $20 \mu \mathrm{M}$ furanone inhibited QS-dependent swarming motility in Serratia liquefaciens, a model QS reporter (39). It has been hypothesized that Delisea sp. utilizes QS interference as a defense mechanism to block formation of bacterial communities on its surface (biofilms).

A second example of eukaryotic signal interference is the observed degradation of the $P$. aeruginosa AHL signal 3-oxo$\mathrm{C}_{12} \mathrm{HSL}$ by human airway epithelial cells (6). Interestingly, specific AHLs such as $\mathrm{C}_{6} \mathrm{HSL}$ and 3-oxo- $\mathrm{C}_{12} \mathrm{HSL}$ were degraded rapidly by epithelial cells, whereas other AHLs, such as 3-oxo$\mathrm{C}_{6} \mathrm{HSL}$, were not degraded rapidly. Recently, Ozer et al. (31) demonstrated that mammalian serum contains paraoxonase, an AHL lactonase that degrades 3 -oxo- $\mathrm{C}_{12} \mathrm{HSL}$. Paraoxonase, encoded by the PON1 gene, is a member of a family of mammalian lactonases that share a high sequence similarity but have distinct patterns of expression and substrate specificities. Mouse serum effectively degraded 3-oxo- $\mathrm{C}_{12} \mathrm{HSL}$ and blocked biofilm development in vitro by $P$. aeruginosa. Surprisingly, PON1-Knockout mice, unable to produce this lactonase, survived $P$. aeruginosa infection at higher levels than expected. It was observed that in PON1-KO mice, the levels of two other members of this family of lactonases, PON2 and PON3, were elevated. It is proposed that paraoxonase-mediated AHL signal degradation may serve as a mechanism of innate resistance by mammals to bacterial infections.

A third mechanism of QS interference is via the destabilization of the R protein. A screen of 50 isolates of Penicillium spp. using two quorum sensing inhibition (QSI) selection strains indicated that $33 \%$ of these isolates were able to block QS regulation (40). These QSI strains contained plasmids in which QS promoters are fused to genes encoding toxic metabolites. Therefore, QSI strain growth can only occur in the presence of inhibitors that block normal QS-dependent promoter expression. Two of the Penicillium compounds were identified as penicillic acid and patulin. Patulin, a polyketide lactone, was originally identified as a mycotoxin that can be present in apple juice (Fig. 1C) (14). Addition of patulin to an Escherichia coli strain that over-produced LuxR caused a significant reduction in detectable LuxR protein as determined by fluorescent antibody detection $(23,40)$. This $\mathrm{R}$ protein degradation was hypothesized to be a fungal defense against QS-dependent bacterial attackers. 
Plant-bacterial communication. Communication between plants and bacteria has been studied extensively in the Rhizobium spp.-legume symbiotic association (3). Studies have documented the exquisite signal communication that occurs by both the bacteria and the plant, resulting in colonization, infection, nodule development, and nitrogen fixation. Communication between the plant pathogen Agrobacterium tumefaciens and its hosts also has been studied extensively (3). However, recent work suggests that interkingdom communication between plant hosts and associated microbes may be widespread, and appears to occur bi-directionally. For example, many plant hosts are capable of enhancing or interfering with bacterial communication systems and several bacterial signal molecules have been shown to influence plant gene expression patterns (described below). Together, these results suggest that understanding this interkingdom communication may lead to reduced disease and improved biological control.

Plant signal mimics. Teplitski et al. (43) demonstrated that plants produced compounds that interfered with and activated QS regulation. In this work, the production of the QS-dependent purple pigment violacein by Chromobacterium violaceum CV026 was inhibited by the presence of pea seedlings in vitro. Strain CV026 is defective in production of the AHL signal $\mathrm{C}_{4} \mathrm{HSL}$ and can only produce violacein if exogenous $\mathrm{C}_{4} \mathrm{HSL}$ is added. When pea seedlings were placed on a lawn of CV026 in the presence of $\mathrm{C}_{4} \mathrm{HSL}$, white zones appeared surrounding the roots. These patches represented areas where root compounds inhibited QS activation of violacein production by strain CV026. Interestingly, pea roots also were shown to activate QS-dependent genes. Escherichia coli strains containing reporter plasmids were constructed in which bioluminescence was dependent on activation by three different $\mathrm{R}$ regulatory proteins (LuxR, AhyR, or LasR). In the absence of AHL signals, bioluminescence was low. In contrast, the presence of pea seedlings resulted in higher levels of bioluminescence. These data suggest that plants have the capacity to both interfere with and activate bacterial QS-dependent gene expression.

Bacterial signals affect eukaryotic metabolism. The idea that plants listen and respond to bacterial communication signals is suggested by the work of Mathesius et al. (26). The roots of Medicago truncatula seedlings were sprayed with a solution of 3oxo- $\mathrm{C}_{12}$ HSL or 3-oxo- $\mathrm{C}_{16: 1} \mathrm{HSL}$. Proteomic analysis of total root extracts indicated that the accumulation of a number of plant proteins $(>150)$ was differentially affected by the presence of AHL signals. Of particular interest, a number of the proteins appear to be involved in plant defense. The effect of bacterial AHLs on plant gene expression was further quantified using transgenic $M$. truncatula plants containing an auxin-responsive promoter and several chalcone synthase promoters fused to uidA encoding $\beta$ glucuronidase. The presence of $50 \mu \mathrm{M} \mathrm{C}{ }_{12} \mathrm{HSL}$ resulted in large increases in $\beta$-glucuronidase expression, confirming that the AHL signal addition influenced plant gene expression.

Work using mammalian cell cultures indicated that AHLs also influenced mammalian gene expression (46). Transgenic mammalian cells were constructed in which a QS-dependent promoter was fused to a nuclear localization signal (NLS) connected to GFP. Without AHL addition, GFP fluorescence was visible only in the cytoplasm. In contrast, after addition of AHL, all GFP fluorescence was rapidly localized to the nucleus. These reports are consistent with the hypothesis that AHLs directly influence gene expression patterns within eukaryotic host tissues.

Potential use of bacterial signaling to improve disease control. Can our increased understanding of bacterial communication be used to improve our ability to control plant pathogens in agriculture? The answer is yes! This potential is illustrated by the following examples. Bacillus thuringiensis was first identified as

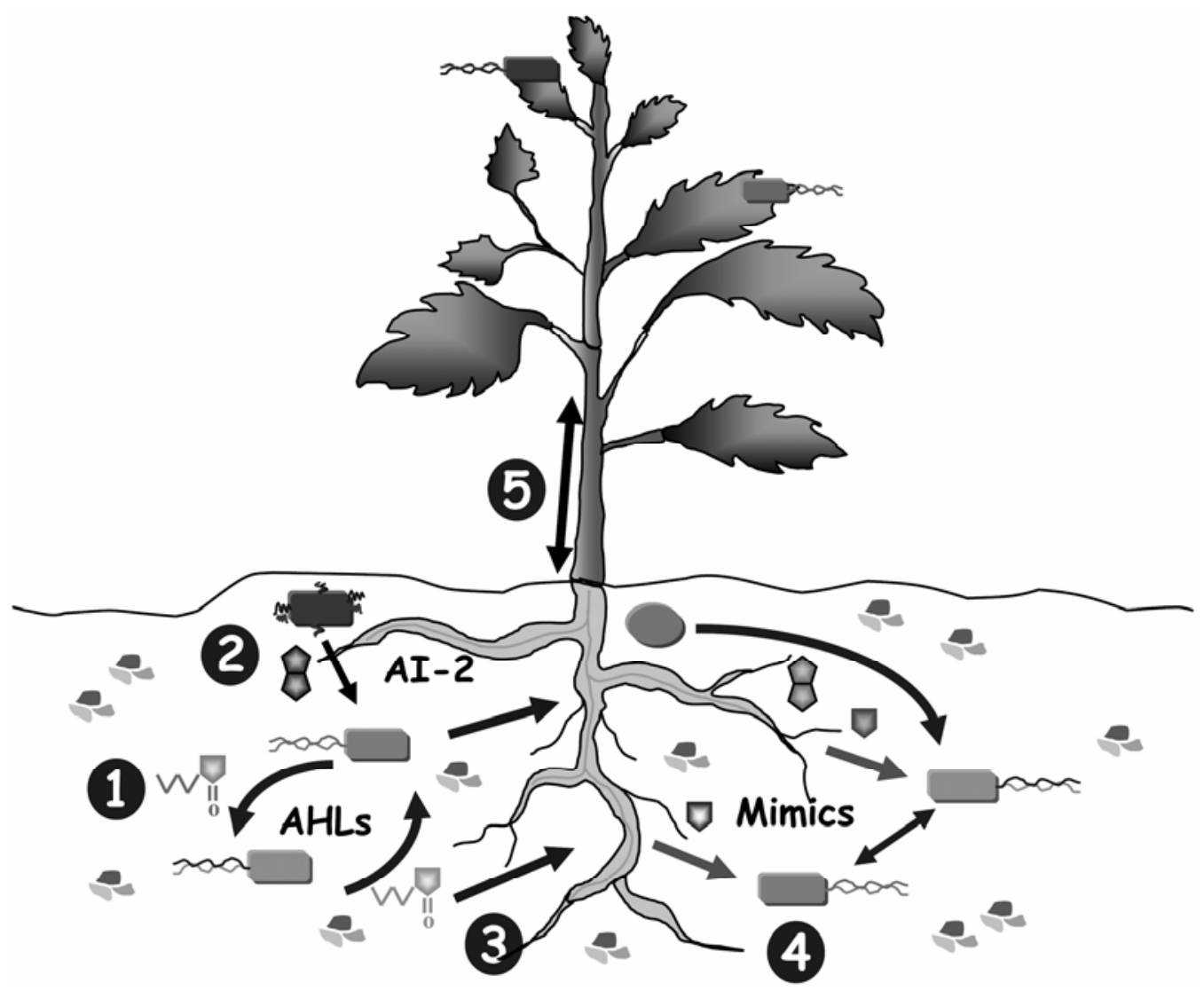

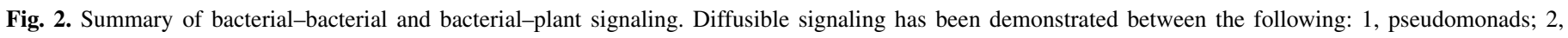

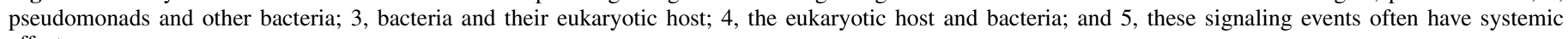
effects. 
a promising biological control agent against insect pathogens due to its ability to produce insecticidal toxins (Bt toxins). The recent discovery that Bacillus and other bacteria produce AHLdegrading enzymes suggests that they also may be useful against QS-utilizing pathogenic bacteria. Dong et al. (12) showed that application of $B$. thuringiensis to potato tubers resulted in inhibition of QS-regulated soft-rot symptom development caused by $E$. carotovora. The net result was control of E. carotovora by the AHL-degrading Bacillus sp., presumably through interference with the pathogen's ability to express QS-dependent virulence traits.

Fray et al. (18) approached this concept from a different perspective. Tobacco plants were engineered to express the Yersinia enterocolitica yenI AHL synthase gene in order to examine the effect of plant AHL production on the expression of genes required for biological control by a plant-associated bacterium. The biological control bacterium P. chlororaphis strain 30-84, as mentioned previously, produces phenazine antibiotics under PhzR/PhzI QS control that inhibit the fungal pathogen of wheat, Gaeumannomyces graminis var. tritici (36). A strain 30-84 phzI mutant, defective in AHL production, produces no phenazines and is no longer inhibitory to the fungal pathogen (48). Addition of extracts of the yenI-engineered tobacco plants restored phenazine production and fungal inhibition, whereas extracts of control plants that did not express yenI did not restore phenazine production or pathogen inhibition (18). Thus, the production of bacterial AHL signals by the plant host complemented an $\mathrm{AHL}^{-}$biological control bacterium and restored its ability to inhibit a plant pathogen.

One of the most promising experiments demonstrated that engineering plants to degrade AHL signals effectively blocked bacterial communication required for pathogenicity $(10,11)$. Tobacco and potato plants were engineered to express the Bacillus aiiA AHL lactonase enzyme and then challenged with E. carotovora. The engineered leaves and tubers showed little if any pathogen damage, whereas the nontransgenic control plants had severe softrot symptoms. This interference with QS-regulated pathogenicity gene activation is functionally analogous to the interference of $P$. aeruginosa biofilm development by paraoxonase-mediated AHL degradation in mammalian serum (31). Together, these experiments indicate that interfering with or augmenting bacterial communication channels can have profound results on the outcome of plant-bacterial interactions, both beneficial and pathogenic.

Summary and concluding thoughts. Communication between and among bacterial populations, once considered an interesting and novel observation, is now recognized as widespread. Communication between microbes and their hosts is known to affect directly the expression of bacterial traits critical to all aspects of bacterial-host interactions (Fig. 2). Examples of bacterial communication include those that occur between members of a single population or between members of different bacterial populations. Mechanisms of communication between bacteria and eukaryotic hosts such as fungi, algae, plants, and animals also have been identified. The influence of this communication network on gene expression patterns and the development of plant-microbe interactions are just beginning to be understood. Additionally, QS signaling appears to be a two-way street in that the eukaryotic host can produce QS signal mimics that directly influence the expression of bacterial genes involved in hostmicrobe interactions, and bacterial QS signals also can influence eukaryotic host gene expression patterns. These communication networks represent key ecological control points that play a key role in the outcome of host-microbe interactions. Understanding these communication networks represents an exciting area of research, and may facilitate large-scale improvements in bacterial-host interactions, pathogen suppression, and biological control.

\section{ACKNOWLEDGMENTS}

We thank K. Maddula and G. Puopolo for critical review of the manuscript. Some of the laboratory work was supported by NRICGP grant 01-02684.

\section{LITERATURE CITED}

1. Ahmer, B. M. 2004. Cell-to-cell signaling in Escherichia coli and Salmonella enterica. Mol. Microbiol. 52:933-945.

2. Bassler, B. L., Greenberg, E. P., and Stevens, A. M. 1997. Cross-species induction of luminescence in the quorum-sensing bacterium Vibrio harveyi. J. Bacteriol. 179:4043-4045.

3. Brencic, A., and Winans, S. C. 2005. Detection of and response to signals involved in host-microbe interactions by plant-associated bacteria. Microbiol. Mol. Biol. Rev. 69:155-194.

4. Bredenbruch, F., Nimtz, M., Wray, V., Morr, M., Muller, R., and Haussler, S. 2005. Biosynthetic pathway of Pseudomonas aeruginosa 4-hydroxy-2alkylquinolines. J. Bacteriol. 187:3630-3635.

5. Cao, H., Krishnan, G., Goumnerov, B., Tsongalis, J., Tompkins, R., and Rahme, L. G. 2001. A quorum sensing-associated virulence gene of Pseudomonas aeruginosa encodes a LysR-like transcription regulator with a unique self-regulatory mechanism. Proc. Natl. Acad. Sci. 98:14613-14618.

6. Chun, C. K., Ozer, E. A., Welsh, M. J., Zabner, J., and Greenberg, E. P. 2004. Inactivation of a P.aeruginosa QS signal by human airway epithelia. Proc. Natl. Acad. Sci. 101:3587-3590.

7. Diggle, S. P., Cornelis, P., Williams, P., and Camara, M. 2006. 4quinolone signaling in Pseudomonas aeruginosa: Old molecules, new perspectives. Int. J. Med. Microbiol. 296:83-91.

8. Diggle, S. P., Winzer, K., Chhabra, S. R., Worrall, K. E., Camara, M., and Williams, P. 2003. The Pseudomonas aeruginosa quinolone signal molecule overcomes the cell density-dependency of the quorum sensing hierarchy, regulates $r h l$-dependent genes at the onset of stationary phase and can be produced in the absence of LasR. Mol. Microbiol. 50:29-43.

9. Dong, Y. H., Gusti, A. R., Zhang, Q., Xu, J., and Zhang, L. H. 2002. Identification of quorum-quenching $N$-acyl homoserine lactonases from Bacillus species. Appl. Environ. Microbiol. 68:1754-1759.

10. Dong, Y. H., Wang, L. H., Xu, J. L., Zhang, H. B., Zhang, X. F., and Zhang, L. H. 2001. Quenching quorum-sensing-dependent bacterial infection by an $N$-acyl homoserine lactonase. Nature 411:813-817.

11. Dong, Y. H., Xu, J. L., Li, X. Z., and Zhang, L. H. 2000. AiiA, an enzyme that inactivates the acylhomoserine lactone quorum-sensing signal and attenuates the virulence of Erwinia carotovora. Proc. Natl. Acad. Sci. 97:3526-3531.

12. Dong, Y. H., Zhang, X. F., Xu, J. L., and Zhang, L. H. 2004. Insecticidal Bacillus thuringiensis silences Erwinia carotovora virulence by a new form of microbial antagonism, signal interference. Appl. Environ. Microbiol. 70:954-960.

13. Dong, Y. H., and Zhang, L. H. 2005. Quorum sensing and quorumquenching enzymes. J. Microbiol. 43:101-109.

14. Drusch, S., and Ragab, W. 2003. Mycotoxins in fruits, fruit juices, and dried fruits. J. Food Prot. 66:1514-1527.

15. Duan, K., Dammel, C., Stein, J., Rabin, H., and Surette, M. G. 2003. Modulation of $P$. aeruginosa gene expression by host microflora through interspecies communication. Mol. Microbiol. 50:1477-1491.

16. Dunlap, P. V. 1999. Quorum regulation of luminescence in Vibrio fischeri. J. Mol. Microbiol. Biotechnol. 1:5-12.

17. Eberl, L. 1999. N-Acyl homoserinelactone-mediated gene regulation in gram-negative bacteria. Syst. Appl. Microbiol. 22:493-506.

18. Fray, R. G., Throup, J. P., Daykin, M., Wallace, A., Williams, P., Stewart, G. S. A. B., and Grierson, D. 1999. Plants genetically modified to produce AHLs communicate with bacteria. Nature Biotech. 17:1017-1020.

19. Fuqua, C., Parsek, M. R., and Greenberg, E. P. 2001. Regulation of gene expression by cell-to-cell communication: Acyl-homoserine lactone quorum sensing. Ann. Rev. Genet. 35:439-468.

20. Guina, T., Purvine, S. O., Yi, E. C., Eng, J., Goodlett, D. R., Aebersold, R., and Miller, S. I. 2003. Quantitative proteomic analysis indicates increased synthesis of a quinolone by Pseudomonas aeruginosa isolates from cystic fibrosis airways. Proc. Natl. Acad. Sci. 100:2771-2776.

21. Huang, J. J., Han, J. I., Zhang, L. H., and Leadbetter, J. R. 2003. Utilization of AHL quorum signals for growth by a soil pseudomonad and P. aeruginosa PAO1. Appl. Environ. Microbiol. 69:5941-5949.

22. Lin, Y. H., Xu, J. L., Wang, L. H., Ong, S. L., Leadbetter, J. R., and Zhang, L. H. 2003. Acyl-homoserine lactone acylase from Ralstonia strain XJ12B represents a novel and potent class of quorum-quenching enzymes. Mol. Microbiol. 47:849-860.

23. Manefield, M., Rasmussen, T. B., Henzter, M., Andersen, J. B., Steinberg, P., Kjelleberg, S., and Givskov, M. 2002. Halogenated furanones inhibit 
quorum sensing through accelerated LuxR turnover. Microbiology 148: 1119-1127

24. Manefield, M., Welch, M., Givskov, M., Salmond, G. P., and Kjelleberg, S. 2001. Halogenated furanones from the red alga, Delisea pulchra, inhibit carbapenem antibiotic synthesis and exoenzyme virulence factor production in the phytopathogen Erwinia carotovora. FEMS Microbiol. Lett. 205:131-138.

25. Mashburn, L. M., and Whiteley, M. 2005. Membrane vesicles traffic signals and facilitate group activities in a prokaryote. Nature 437:422425.

26. Mathesius, U., Mulders, S., Gao, M., Teplitski, M., Caetano-Anollés, G., Rolfe, B. G., and Bauer, W. D. 2003. Extensive and specific responses of a eukaryote to bacterial quorum-sensing signals. Proc. Natl. Acad. Sci. 100:1444-1449.

27. Mavrodi, D. V., Ksenzenko, V. N., Bonsall, R. F., Cook, R. J., Boronin, A. M., and Thomashow, L. S. 1998. A seven-gene locus for synthesis of phenazine-1-carboxylic acid by Pseudomonas fluorescens 2-79. J. Bacteriol. 180:2541-2548.

28. McGrath, S., Wade, D. S., and Pesci, E. C. 2004. Dueling quorum sensing systems in Pseudomonas aeruginosa control the production of the Pseudomonas quinolone signal (PQS). FEMS Microbiol. Lett. 230:27-34.

29. Morello, J., Pierson, E. A., and Pierson, L. S. 2004. Negative crosscommunication among wheat rhizosphere bacteria: Effect on antibiotic production by the biological control bacterium $P$. aureofaciens 30-84. Appl. Environ. Microbiol. 70:3103-3109.

30. Nealson, K. H., Platt, T., and Hastings, J. W. 1970. Cellular control of the synthesis and activity of the bacterial luminescence system. J. Bacteriol. 104:313-322.

31. Ozer, E. A., Pezzulo, A., Shih, D. M., Chun, C., Furlong, C., Lusis, A. J., Greenberg, E. P., and Zabner, J. 2005. Human and murine paraoxonase 1 are host modulators of Pseudomonas aeruginosa quorum-sensing. FEMS Microbiol Lett. 253:29-37.

32. Pesci, E. C., Milbank, J. B., Pearson, J. P., McKnight, S., Kende, A. S., Greenberg, E. P., and Iglewski, B. H. 1999. Quinolone signaling in the cell-to-cell communication system of Pseudomonas aeruginosa. Proc. Nat. Acad. Sci. 96:11229-11234.

33. Pierson, E. A., Wood, D. W., Cannon, J. A., Blachere, F. M., and Pierson, L. S., III. 1998. Interpopulation signaling via $N$-acyl-homoserine lactones among bacteria in the wheat rhizosphere. Mol. Plant-Microbe Interact. 11:1078-1084.

34. Pierson, L. S., III, Keppenne, V. D., and Wood, D. W. 1994. Phenazine antibiotic biosynthesis in Pseudomonas aureofaciens 30-84 is regulated by PhzR in response to cell density. J. Bacteriol. 176:3966-3974.

35. Pierson, L. S., III, Lam, S., Gaffney, T., and Gong, F. C. 1995. Molecular analysis of genes encoding phenazine biosynthesis in the biological control bacterium Pseudomonas aureofaciens 30-84. FEMS Microbiol. Lett. 134:299-307.

36. Pierson, L. S., III, and Thomashow, L. S. 1992. Cloning and heterologous expression of phenazine biosynthetic locus from Pseudomonas aureofaciens 30-84. Mol. Plant-Microbe Interact. 5:330-339.

37. Pierson, L. S., III, Wood, D. W., and Pierson, E. A. 1998. Homoserine lactone-mediated gene regulation in plant-associated bacteria. Annu. Rev.
Phytopathol. 36:207-225.

38. Qin, Y., Luo, Z. Q., Smyth, A. J., Gao, P., von Bodman, S. B., and Farrand, S. K. 2000. Quorum-sensing signal binding results in dimerization of TraR and its release from membranes into the cytoplasm. EMBO J. 19:5212-5221.

39. Rasmussen, T., Manefield, M., Andersen, J. B., Eberl, L., Anthoni, U., Christophersen, C., Steinberg, P., Kjelleberg, S., and Givskov, M. 2000. How D. pulchra furanones affect quorum sensing and swarming motility in S. liquefaciens MG. Microbiology 146:3237-3244.

40. Rasmussen, T. B., Skindersoe, M. E., Bjarnsholt, T., Phipps, R. K., Christensen, K. B., Jensen, P. O., Andersen, J. B., Koch, B., Larsen, T. O., Hentzer, M., Eberl, L., Hoiby, N., and Givskov, M. 2005. Identity and effects of quorum-sensing inhibitors produced by Penicillium species. Microbiology 151:1325-1340.

41. Steidle, A., Sigl, K., Schuhegger, R., Ihring, A., Schmid, M., Gantner, S., Stoffels, M., Riedel, K., Givskov, M., Hartmann, A., Langebartels, C., and Eberl, L. 2001. Visualization of AHL-mediated cell-cell communication between bacteria colonizing the tomato rhizosphere. Appl. Environ. Microbiol. 67:5761-5770.

42. Surette, M. G., Miller, M. B., and Bassler, B. L. 1999. Quorum sensing in Escherichia coli, Salmonella typhimurium, and Vibrio harveyi: A new family of genes responsible for autoinducer production. Proc. Natl. Acad. Sci. 96:1639-1644

43. Teplitski, M., Robinson, J. B., and Bauer, W. D. 2000. Plants secrete substances that mimic bacterial AHL signal activities and affect population density-dependent behaviors in associated bacteria. Mol. Plant-Microbe Interact. 13:637-648.

44. Vendeville, A., Winzer, K., Heurlier, K., Tang, C. M., and Hardie, K. R. 2005. Making sense of metabolism: Autoinducer-2, LuxS and pathogenic bacteria. Nature Rev. 3:383-396.

45. Wang, Y. J., and Leadbetter, J. R. 2005. Rapid acyl-homoserine lactone quorum signal biodegradation in diverse soils. Appl. Environ. Microbiol. 71:1291-1299.

46. Williams, S. C., Patterson, E. K., Carty, N. L., Griswold, J. A., Hamood, A. N., and Rumbaugh, K. P. 2004. P. aeruginosa AI enters and functions in mammalian cells. J. Bacteriol. 186:2281-2287.

47. Williamson, L. L., Borlee, B. R., Schloss, P. D., Guan, C., Allen, H. K., and Handelsman, J. 2005. Intracellular screen to identify metagenomic clones that induce or inhibit a quorum-sensing biosensor. Appl. Environ. Microbiol. 71:6335-6344.

48. Wood, D. W., and Pierson, L. S., III. 1996. The phzI gene of Pseudomonas aureofaciens 30-84 is responsible for the production of a diffusible signal required for phenazine antibiotic production. Gene 168:49-53.

49. Xavier, K. B., and Bassler, B. L. 2003. LuxS quorum sensing: More than just a numbers game. Curr. Opin. Microbiol. 6:191-197.

50. Xavier, K. B., and Bassler, B. L. 2005. Interference with AI-2-mediated bacterial cell-cell communication. Nature 437:750-753

51. Zhu, J., and Winans, S. C. 1999. Autoinducer binding by the quorumsensing regulator TraR increases affinity for target promoters in vitro and decreases TraR turnover rates in whole cells. Proc. Natl. Acad. Sci. 96:4832-4837. 